\title{
Die meistzitierten Arbeiten im 37. Jahrgang der Psychiatrischen Praxis - Herzlichen Glückwunsch!
}

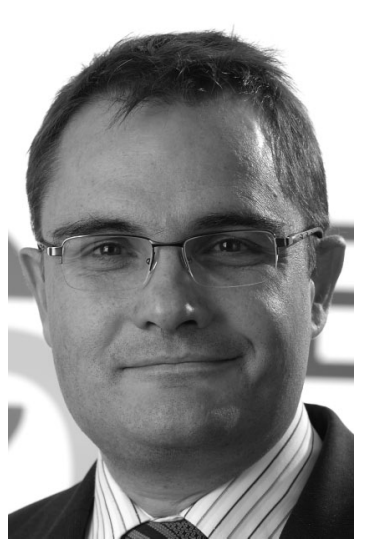

Isaac Bermejo

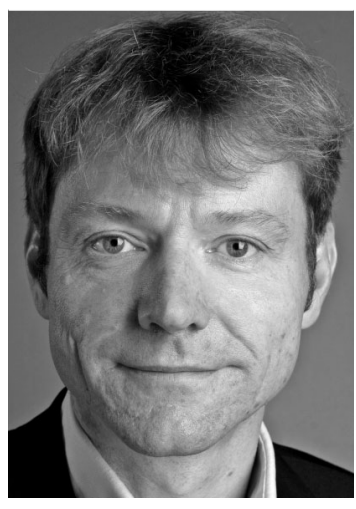

Hans-Helmut König

\section{Versorgungsrelevante Themen}

$\nabla$

Auch in diesem Jahr küren wir wieder die meistzitierten Arbeiten der Psychiatrischen Praxis und können zwei gleichrangige Arbeiten prämieren. Die Arbeiten greifen wichtige versorgungsrelevante Themen auf: Psychische Störungen bei Menschen mit Migrationshintergrund und neue Finanzierungsmodelle der Versorgung.

\section{Psychische Gesundheit bei Migranten $\nabla$}

Isaac Bermejo aus Freiburg und die Arbeitsgruppe um Martin Härter, Universitätsklinikum Hamburg-Eppendorf, analysierten die Daten des Bundesgesundheitssurveys und verglichen die Häufigkeit von psychischen Störungen bei Menschen mit Migrationshintergrund mit der Häufigkeit psychischer Störungen in der deutschen Allgemeinbevölkerung. Sie konnten zeigen, dass Menschen mit Migrationshintergrund signifikant höhere Prävalenzraten psychischer Störungen aufwiesen. Dies traf besonders auf somatoforme und affektive Störungen zu. Sie verwiesen auf die Notwendigkeit einer migrantensensiblen epidemiologischen Forschung und diskutierten die Konsequenzen für eine adäquate psychiatrische Versorgung [1].

\section{Das regionale Psychiatriebudget $\nabla$}

Schon länger wird darüber nachgedacht, wie mit neuen Finanzierungsmodellen und verändertem Anreiz die Strukturen für die Leistungsanbieter für eine effizientere Versorgung der Patienten gefördert werden können. Hans-Helmut König, Professor für Gesundheitsökonomie am Universitätsklinikum Hamburg-Eppendorf, legt eine Analyse zu den langfristigen Auswirkungen eines regionalen Psychiatriebudgets vor. Dabei wurden in einer prospektiven Kohortenstudie 258 Patienten einer Modellregion mit regionalem Psychiatriebudget (Itzehoe/Steinburg) mit 244 Patienten aus einer
Kontrollregion mit psychiatrischer Standardversorgung (Kreis Dithmarschen) verglichen. Es konnte gezeigt werden, dass das regionale Psychiatriebudget es psychiatrischen Kliniken erlaubt, Versorgungsangebote mit höherer Flexibilität und personeller Kontinuität zu unterbreiten. Dies schlug sich in einem verbesserten Funktionsniveau in der Modellregion nieder. Im Hinblick auf die Kosten kam es durch diesen Ansatz zu Verschiebungen zwischen den verschiedenen Behandlungssettings. Durch das regionale Psychiatriebudget konnten zwar die Gesamtkosten psychiatrischer Versorgung nicht reduziert werden, aber das Budget bot den Leistungsanbietern und Kostenträgern Planungssicherheit [2].

Die aufgegriffenen Themen werden auch weiterhin in der Psychiatrischen Praxis aktiv diskutiert [3-5].

Wir gratulieren ganz herzlich und wünschen den Preisträgern alles Gute!

\section{Steffi Riedel-Heller}

\section{Literatur}

1 Bermejo I, Mayninger E, Kriston L et al. Psychische Störungen bei Menschen mit Migrationshintergrund im Vergleich zur deutschen Allgemeinbevölkerung. Psychiat Prax 2010; 37: 225-232

2 König $H-H$, Heinrich $S$, Heider $D$ et al. Das Regionale Psychiatriebudget (RPB): Ein Modell für das neue pauschalierende Entgeltsystem psychiatrischer Krankenhausleistungen? Psychiat Prax 2010; 37: $34-42$

3 Lauber C. Krankenversorgung - Versorgungsplanung - Versorgungspolitik - Versorgungsforschung. Psychiat Prax 2012; 39: 1 -2

4 Bermejo I, Frank F, Maier I et al. Gesundheitsbezogenes Inanspruchnahmeverhalten von Personen mit Migrationshintergrund und einer psychischen Störung im Vergleich zu Deutschen. Psychiat Prax 2012; 39: $64-70$

5 Bermejo I, Nicolaus L, Kriston L et al. Vergleichende Analyse psychosomatischer Beschwerden bei Personen mit spanischem, italienischem, türkischem und russischem Migrationshintergrund. Psychiat Prax 2012; 39: $157-163$ 10.1055/s-0032-1327417

Psychiat Prax 2013; 40: 7

(c) Georg Thieme Verlag KG

Stuttgart · New York

ISSN 0303-4259

Korrespondenzadresse

Prof. Dr. med.

Steffi G. Riedel-Heller, MPH

Institut für Sozialmedizin,

Arbeitsmedizin und

Public Health (ISAP),

Universität Leipzig

Philipp-Rosenthal-Straße 55

04103 Leipzig

Steffi.Riedel-Heller@medizin.

uni-leipzig.de 